\title{
Nonlinear spectroscopy of photons bound to one atom
}

\author{
I. SCHUSTER, A. KUBANEK, A. FUHRMANEK, T. PUPPE, P. W. H. PINKSE, K. MURR AND G. REMPE* \\ Max-Planck-Institut für Quantenoptik, Hans-Kopfermann-Straße 1, D-85748 Garching, Germany \\ *e-mail: gerhard.rempe@mpq.mpg.de
}

Optical nonlinearities typically require macroscopic media, thereby making their implementation at the quantum level an outstanding challenge. Here, we demonstrate a nonlinearity for one atom enclosed by two highly reflecting mirrors ${ }^{1}$. We send laser light through the input mirror and record the light from the output mirror of the cavity. For weak laser intensity, we find the vacuum-Rabi resonances ${ }^{2-11}$. But for higher intensities, we observe an extra resonance ${ }^{12}$, which originates from the fact that the cavity can accommodate only an integer number of photons and that this photon number determines the characteristic frequencies of the coupled atom-cavity system ${ }^{13-15}$. We selectively excite such a frequency by depositing two photons at once into the system and find a transmission that increases with the laser intensity squared. The nonlinearity differs from classical saturation nonlinearities ${ }^{16-19}$ and is direct spectroscopic proof of the quantum nature of the atom-cavity system. It provides a photon-photon interaction by means of one atom, and constitutes a step towards a two-photon gateway or a singlephoton transistor ${ }^{20}$.

The quantum nonlinearity has its origin in the fact that under the condition of strong coupling, a system composed of a single atom and a single cavity mode has properties that are distinctively different from those of the bare atom (without the cavity), or the bare cavity (without the atom), or just the sum of the two (Fig. 1a). In fact, the composite system forms a new quantum entity, the so-called atom-cavity molecule, made of matter and light, with its own characteristic energy spectrum. This spectrum consists of an infinite ladder of pairs of states, the dressed states (Fig. 1b). The first doublet contains one quantum of energy and can be probed by laser spectroscopy. For weak probing, the resulting spectrum is independent of the laser intensity and has been dubbed the vacuum-Rabi or normal-mode spectrum, consisting of a pair of resonances symmetrically split around the bare atomic and cavity resonances. This spectrum was first observed with atomic beams ${ }^{1}$, and has been explored recently with single dipole-trapped atoms $\mathrm{s}^{2-4}$. It constitutes a benchmark for strong atom-cavity coupling and is central to most cavity quantum electrodynamics (QED) experiments, including those outside atomic physics ${ }^{5-11}$. Note that the normal-mode spectrum on its own can equally well be described classically, by linear dispersion theory or a coupled oscillator model (atomic dipole and cavity field).

The next-higher-lying doublet contains two quanta of energy and lacks a classical explanation ${ }^{12,21,22}$. The corresponding dressed states have been observed (together with a few higher-order states) in microwave cavity $\mathrm{QED}^{13-15}$ and even ion trapping, where phonons play the role of photons ${ }^{23}$. At optical frequencies, evidence for these states has indirectly been obtained in two-photon correlation experiments where the conditional response of the system on detection of an emitted photon is monitored ${ }^{16,24-26}$. These optical experiments observe the quantum fluctuations in dissipative cavity QED systems but operate away from a resonance to a higher-lying state. Direct spectroscopy using a two-colour technique to excite the second doublet step-wise has been attempted in a pioneering experiment with atomic beams ${ }^{22}$. An unambiguous signature of these states remained elusive owing to large fluctuations in the number of atoms traversing the cavity.

Using single trapped atoms, we exploit the anharmonicity of the energy-level spectrum to drive a multiphoton transition directly from the vacuum state to a specific higher-lying state. We observe the quantum character of our cavity QED field by measuring a photon flux, not a photon correlation. To explain our technique, we note that a two-state atom coupled to a single-mode light field has a discrete spectrum consisting of a ladder of dressed states, $|n+1, \mp\rangle$, with frequencies

$$
\omega_{n+1, \mp}=n \omega_{\mathrm{c}}+\frac{1}{2}\left(\omega_{\mathrm{a}}+\omega_{\mathrm{c}}\right) \mp \frac{1}{2} \sqrt{4 g^{2}(n+1)+\left(\omega_{\mathrm{a}}-\omega_{\mathrm{c}}\right)^{2}}
$$

and a ground state $|g, 0\rangle$ with zero energy (atom with ground state $|g\rangle$ and mode in the vacuum state $|0\rangle)$. Here, $n=0,1,2, \ldots$ is the principal quantum number of the mode (to be distinguished from the mean photon number), $g$ is the atom-cavity coupling strength and $\omega_{\mathrm{a}}$ and $\omega_{\mathrm{c}}$ are the frequencies of the atom and the cavity, respectively. The frequencies of the coupled system are probed with monochromatic light of frequency $\omega_{\mathrm{L}}$ (ref. 12). Resonances occur when $(n+1) \omega_{\mathrm{L}}=\omega_{n+1, \mp}$. In Fig. 1c, these resonance conditions are plotted in the frame $\left(\Delta_{\mathrm{a}}, \Delta_{\mathrm{c}}\right)$ where $\Delta_{\mathrm{a}}=\omega_{\mathrm{L}}-\omega_{\mathrm{a}}$ and $\Delta_{\mathrm{c}}=\omega_{\mathrm{L}}-\omega_{\mathrm{c}}$ are the atom and cavity detunings, respectively.

We now notice that if each laser photon is resonant with the atom, $\omega_{\mathrm{L}}=\omega_{\mathrm{a}}\left(\Delta_{\mathrm{a}}=0\right)$, it is detuned from the single-photon resonances, $\omega_{\mathrm{L}} \neq \omega_{1, \mp}$, and this for any value of the cavity frequency $\omega_{\text {c. }}$. Scanning the cavity frequency (vertical arrow in Fig. 1c) around the frequency of a weak laser therefore gives a suppressed and largely frequency-independent response, as further discussed in connection with Fig. 3. When increasing the laser intensity, however, two-photon transitions can occur at $\Delta_{c}=\mp g$, where two laser photons together are resonantly absorbed by the combined system and the second manifold of dressed states is populated for $2 \omega_{\mathrm{L}}=\omega_{2 \mp}$. We keep the intensity at low enough values such that the atomic transition is never saturated. In this way, we rule out the possibility of nonlinearities due to a classical behaviour of the intracavity field ${ }^{17}$. This protocol of driving multiphoton transitions by avoiding the normal modes as well as atomic saturation is new and should in principle apply to other cavity QED systems. It can be interpreted as a two-photon gateway: single photons 

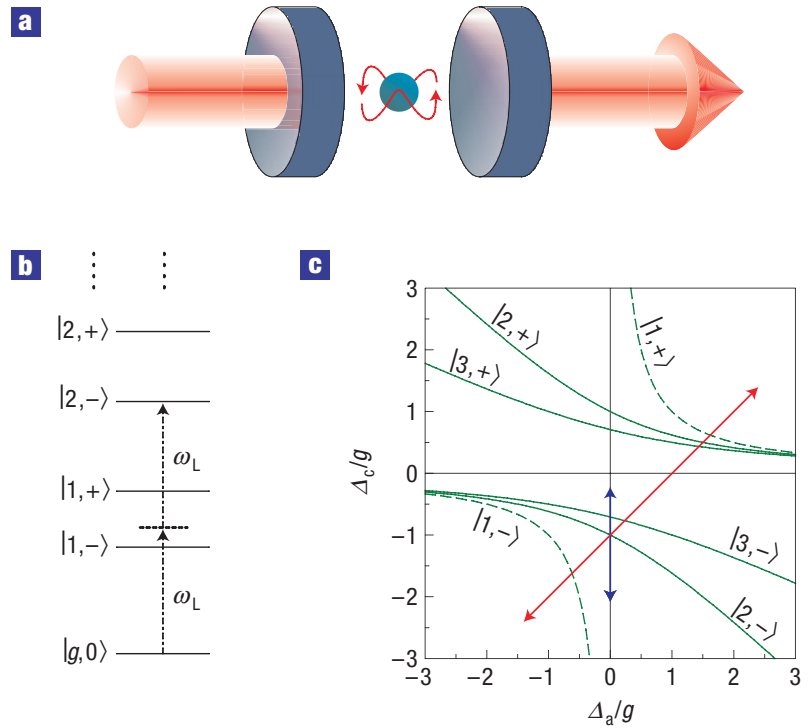

Figure 1 The atom-cavity molecule. a, Artist's conception of an atom enclosed by two cavity mirrors. The coupling of the atom to the intracavity light is so strong that the energy spectrum of the composite atom-cavity 'molecule' radically differs from the spectrum of its components. $\mathbf{b}$, Schematic diagram of such a level structure illustrating how the dressed state $|2,-\rangle$ is directly accessed from the vacuum through a two-photon transition. c, Location of the classical (normal-mode) resonances (dashed curves) and the first two quantum resonances (states $|2, \mp\rangle$ and $|3, \mp\rangle)$ (solid curves). The diagonal arrow is the scan direction shown in Fig. 2, which crosses all resonances. A vertical scan along $\Delta_{\mathrm{a}}=0$, as in Fig. 3 , avoids the classical resonances. Resonances for the manifold $(n+1)$ are located at $\Delta_{\mathrm{c}}= \pm g / \sqrt{n}$ for $\Delta_{\mathrm{a}}=0$.

cannot be accepted by the combined atom-cavity system, but two photons can.

Our implementation of a strongly coupled atom-cavity system consists of single ${ }^{85} \mathrm{Rb}$ atoms localized inside the mode of a high-finesse optical cavity by means of an auxiliary intracavity red-detuned dipole trap at $785 \mathrm{~nm}$. We carry out spectroscopy on the system by shining near-resonant probe light at $780 \mathrm{~nm}$ onto the input mirror and recording the light exiting from the output mirror. While probing the system, we also monitor the localization of the atom. We then post-select only the events for which the condition of strong coupling was fulfilled. For sufficient statistics, we average over many trapping events (see Supplementary Information, Methods).

In a first experiment, the cavity transmission is monitored for an atom-cavity detuning $\Delta_{\mathrm{a}}-\Delta_{\mathrm{c}} \approx g$. For these parameters, the normal-mode spectrum becomes asymmetric and the splitting between states $|1,-\rangle$ and $|2,-\rangle$ increases compared with the case $\Delta_{\mathrm{a}} \approx \Delta_{\mathrm{c}}$. This has the advantage that this two-photon resonance is well separated from the normal modes even for moderate atomcavity coupling constants. Figure 2 shows two scans with different probe laser intensities along the diagonal arrow in Fig. 1c. Both scans show the normal modes, but the higher-intensity scan shows a pronounced extra resonance.

The quantum anharmonicity ${ }^{14}$ of the energy-level structure reveals itself in the position of the two-photon resonance relative to the normal modes, which is in excellent agreement with equation (1) for a coupling of $g=2 \pi \times 11.2 \mathrm{MHz}$ and a Starkshifted atom-cavity detuning of $2 \pi \times 10.5 \mathrm{MHz}$. These values indicate that the coupling is about $70 \%$ of the maximally possible value $g_{0}=2 \pi \times 16 \mathrm{MHz}$ at an antinode, and that the trap induces

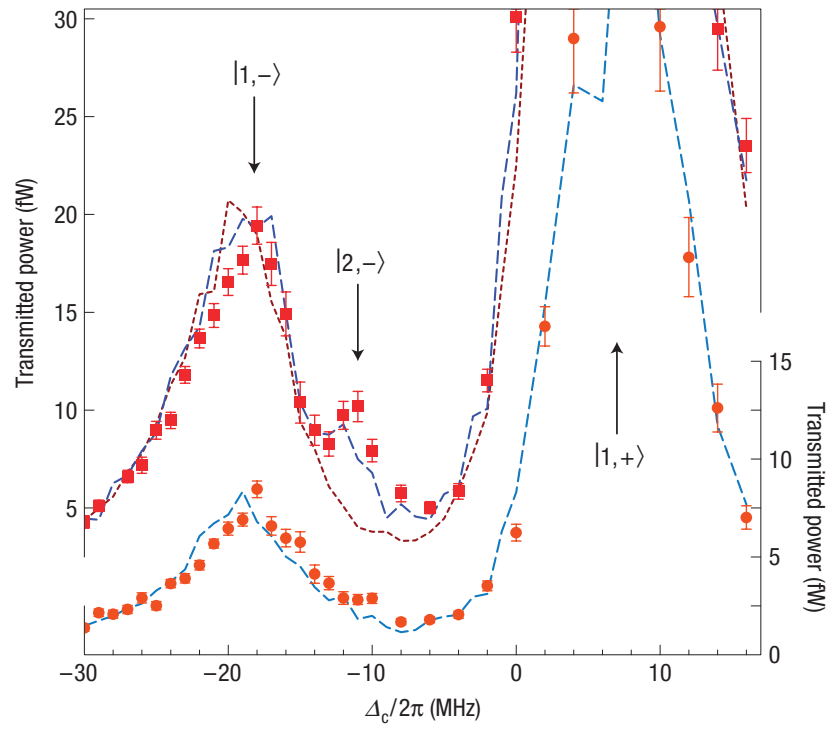

Figure 2 Quantum anharmonicity of the atom-cavity system. Transmitted power as a function of cavity detuning $\Delta_{\mathrm{c}}$ for the input powers $0.5 \mathrm{pW}$ (circles, right scale) and $1.5 \mathrm{pW}$ (squares, left scale). In this and all following figures, $10 \mathrm{fW}$ corresponds to about 0.01 intracavity photons, the error bars are s.d. and an offset of $0.5 \mathrm{fW}$ due to detector dark counts has been removed from the data. The two peaks at $2 \pi \times(-18,7) \mathrm{MHz}$ represent the two normal modes, which are reproduced by a theory considering only single-photon transitions (short-dashed line). Note that the maximum of the right peak is beyond the scale of the figure. The quantization of the intracavity light (long-dashed lines) is required to explain the appearance of the two-photon resonance at $-2 \pi \times 11 \mathrm{MHz}$ at higher intensity.

an average Stark shift of $2 \pi \times 24.5 \mathrm{MHz}$ which also corresponds to about $70 \%$ of the Stark shift at an antinode of the standingwave dipole trap (the trap depth is $170 \mathrm{nW}$ and the bare atomcavity detuning is preset to $2 \pi \times 35 \mathrm{MHz}$ ). Ideal couplings are not reached owing to two reasons: first, the atom performs an oscillation in the dipole trap wells; second, the position of the trapping wells shifts with respect to the antinodes of the probe light along the cavity axis as the distance from the cavity centre increases; therefore, atoms that are trapped slightly off the cavity centre are not maximally coupled.

Next, we carried out extensive numerical simulations to compare the measured spectrum with several cavity QED models ${ }^{12}$. The simulations closely imitate the experiment, starting from the trapping of single, slow atoms which are injected into the intracavity dipole trap, following up with the measurement protocol which is executed until the atom leaves the trap, and culminating in the same evaluation procedure (see Supplementary Information, Discussion). The first set of simulations (only shown for the higher-intensity scan, short-dashed line in Fig. 2) assumes at most one quantum of energy in the system, thereby enabling us to quantify the contribution due to single-photon transitions. We see that, whereas the normal-mode resonances are reproduced, there is a large deviation to the measured data precisely at the position of the two-photon resonance. In contrast, another set of simulations (long-dashed lines) was carried out for a quantized cavity mode with three Fock states, $|0\rangle,|1\rangle,|2\rangle$. Apart from the normal modes, these simulations also reproduce the two-photon resonance.

In a second experiment, we explore the quantum regime by scanning the cavity along the vertical arrow in Fig. 1c, using a bare atom-probe detuning of $2 \pi \times 21 \mathrm{MHz}$ and dipole trap power of $140 \mathrm{nW}$. Figure 3 shows four scans with different probe 


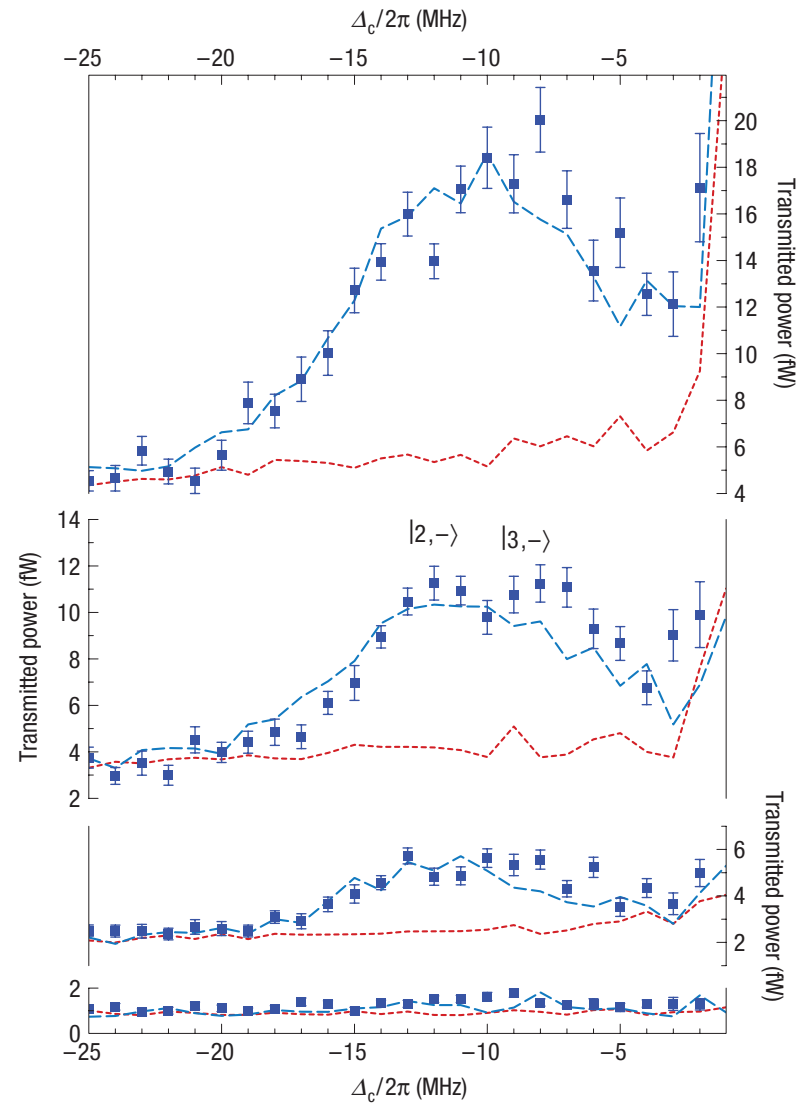

Figure 3 Multiphoton resonances and suppression of single-photon resonances. Transmitted power as a function of cavity detuning $\Delta_{\mathrm{c}}$ for input powers of $0.5,1.5,2.4$ and $3.3 \mathrm{pW}$ (from bottom to top, data points). The simulations with single-photon (short-dashed lines) and multiphoton transitions (long-dashed lines), with the respective positions of the dressed states $|2,-\rangle$ and $|3,-\rangle$ are also shown.

laser intensities. The lowest-intensity scan (bottom panel) shows a largely flat signal, in agreement with our idea of avoiding the normal modes. Here, the photonic state is close to the vacuum state, with a mean intracavity photon number as low as 0.001 . All higherintensity scans, however, show a pronounced extra resonance. The deviation between the off-resonance signal, $\Delta_{c} / 2 \pi \leq-20 \mathrm{MHz}$, and the on-resonance signal, $-15 \mathrm{MHz} \leq \Delta_{\mathrm{c}} / 2 \pi \leq-5 \mathrm{MHz}$, gets larger with increasing intensity. Here, we find that a simulation with at most one quantum of excitation (short-dashed lines) continues to predict a signal with no major modification in the structure, whereas the resonance is globally reproduced with simulations taking into account field quantization. We had to account for four Fock states, indicating that the resonance stems from a twophoton and a weak but rising three-photon transition, the latter transition contributing to broaden the resonance. We also notice an increase in the transmitted intensity at small cavity detunings $\left|\Delta_{c}\right| / 2 \pi \leq 3 \mathrm{MHz}$. This is a systematic effect originating from the bare cavity resonance occurring at $\Delta_{\mathrm{c}}=0$ with a linewidth of $\kappa=2 \pi \times 1.25 \mathrm{MHz}$, which could not be completely suppressed in the post-selection process (see Supplementary Information, Discussion and references therein).

Under coherent excitation, we expect a mainly quadratic scaling of the transmitted intensity at the frequency of the two-photon resonance and a linear scaling off resonance, that is, at large cavity detunings $\left|\Delta_{c}\right| \gg g$, where all theories

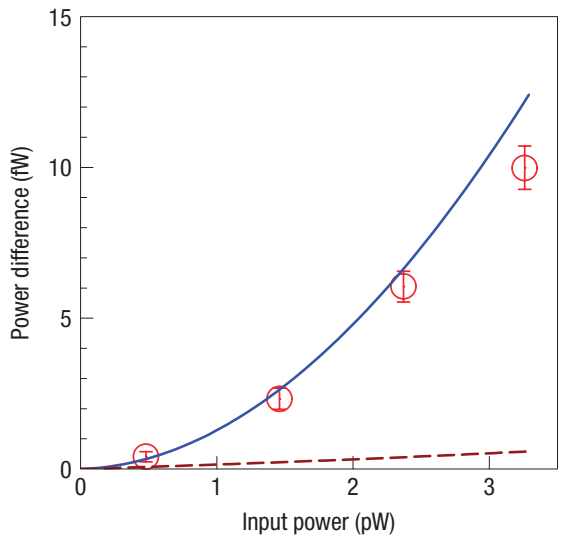

Figure 4 Nonlinear intensity response. Difference between the nonlinear response on the two-photon resonance and the linear single-photon response, together with the prediction from a quantum theory with an immobile atom (solid line). For reference, we also show the nonlinearity expected from the saturation of a two-state atom coupled to a classical field (optical bistability theory, dashed line).

coincide. To evaluate the quantum response of the system, the data used for Fig. 3 are averaged over the two-photon region $\left(-15 \mathrm{MHz} \leq \Delta_{\mathrm{c}} / 2 \pi \leq-10 \mathrm{MHz}\right)$ as well as over the off-resonance region $\left(-25 \mathrm{MHz} \leq \bar{\Delta}_{\mathrm{c}} / 2 \pi \leq-20 \mathrm{MHz}\right)$, for each input intensity. The off-resonant region serves as a reference for the linear single-photon contribution. By taking the difference between the two regions, we isolate the contribution of the two-photon transition, and find a mainly quadratic response of the transmitted intensity versus the input intensity (Fig. 4, circles).

We proceed by comparing the data with a model that assumes the atom to be immobile. This model describes an ideal quantum system, with a well-defined coupling to the mode, as would be desirable for future applications. To this end, we match all of the spectra of Fig. 3 to this idealized theory with a common set of parameters $\left(g, \Delta_{\mathrm{a}}\right)$, and find good agreement for $\left(g, \Delta_{\mathrm{a}}\right)=2 \pi \times(11.5,1) \mathrm{MHz}$ (see Supplementary Information, Fig. S3). Note that the coupling $g$ is close to the value obtained for the independent measurements in Fig. 2, whereas the atomic detuning $\Delta_{\mathrm{a}}$ is close to zero. The theory spectra are then evaluated in the same way as the measured spectra to obtain the intensity response of the system. The resulting nonlinear curve (solid line in Fig. 4) describes the measured data reasonably well and also shows a mainly quadratic dependency. We hope to further approach the fixed-atom limit in our experiment by extending our cooling protocol from one to three dimensions ${ }^{27}$.

For completeness, we also analysed the nonlinear theory of optical bistability ${ }^{16,17}$ and found that it is inconsistent with all of the measurements presented here, as shown with simulations (see Supplementary Information, Discussion and Figs S1,S2). Specifically, the bistability theory predicts a behaviour close to the one we obtained with the theory of coupled oscillators. Explained differently, according to bistability theory, we are operating on the lower branch, where the corresponding nonlinear response is small (dashed line in Fig. 4). Indeed, the reported nonlinearity occurs with an occupation probability of the atomic excited state of at most 0.07. This is what makes it radically different from and dominant over the standard saturation nonlinearity for a two-state atom.

In summary, our experiment enters a new regime, with nonlinear quantum optics at the level of individual atomic and photonic quanta. In the future, we plan to investigate the photon statistics and the spectrum of the light transmitted through the 
cavity. Once improved cooling forces are implemented to better localize the atom in the cavity mode, new multiphoton states could be produced by applying techniques originally developed for a single atom and single photons to the case of a single atomcavity system and multiple photons. Other applications in quantum information science include a single-photon transistor, where one photon controls the propagation of another photon ${ }^{20}$.

Received 10 January 2008; accepted 12 March 2008; published 13 April 2008.

\section{References}

1. Berman, P. R. (ed.) Cavity Quantum Electrodynamics (Advances in Atomic, Molecular, and Optical Physics, Academic, New York, 1994).

2. Boca, A. et al. Observation of the vacuum Rabi spectrum for one trapped atom. Phys. Rev. Lett. $\mathbf{9 3}$ 233603 (2004).

3. Maunz, P. et al. Normal-mode spectroscopy of a single-bound-atom-cavity system. Phys. Rev. Lett. 94, 033002 (2005).

4. Puppe, T. et al. Trapping and observing single atoms in a blue-detuned intracavity dipole trap. Phys. Rev. Lett. 99, 013002 (2007).

5. Wallraff, A. et al. Strong coupling of a single photon to a superconducting qubit using circuit quantum electrodynamics. Nature 431, 162-167 (2004).

6. Reithmaier, J. P. et al. Strong coupling in a single quantum dot-semiconductor microcavity system Nature 432, 197-200 (2004).

7. Yoshie, T. et al. Vacuum Rabi splitting with a single quantum dot in a photonic crystal nanocavity. Nature 432, 200-203 (2004).

8. Peter, E. et al. Exciton-photon strong-coupling regime for a single quantum dot embedded in a microcavity. Phys. Rev. Lett. 95, 067401 (2005).

9. Khitrova, G., Gibbs, H. M., Kira, M., Koch, S. W. \& Scherer, A. Vacuum Rabi splitting in semiconductors. Nature Phys. 2, 81-90 (2006).

10. Press, D. et al. Photon antibunching from a single quantum-dot-microcavity system in the strong coupling regime. Phys. Rev. Lett. 98, 117402 (2007).

11. Hennessy, K. et al. Quantum nature of a strongly coupled single quantum dot-cavity system. Nature 445, 896-899 (2007).

12. Carmichael, H. J., Tian, L., Ren, W. \& Alsing, P. in Cavity Quantum Electrodynamics (ed. Berman, P. R.) 381-423 (Advances in Atomic, Molecular, and Optical Physics, Academic, New York, 1994).

13. Rempe, G., Walther, H. \& Klein, N. Observation of quantum collapse and revival in a one-atom maser. Phys. Rev. Lett. 58, 353-356 (1987).
14. Brune, M. et al. Quantum Rabi oscillation: A direct test of field quantization in a cavity. Phys. Rev. Lett. 76, 1800-1803 (1996).

15. Schuster, D. I. et al. Resolving photon number states in a superconducting circuit. Nature $\mathbf{4 4 5}$ 515-518 (2007).

16. Rempe, G., Thompson, R. J., Brecha, R. J., Lee, W. D. \& Kimble, H. J. Optical bistability and photon statistics in cavity quantum electrodynamics. Phys. Rev. Lett. 67, 1727-1730 (1991).

17. Lugiato, L. A. \& Narducci, L. M. in Fundamental Systems in Quantum Optics, Les Houches, Session LIII, 1990 (eds Dalibard, J., Raimond, J. M. \& Zinn-Justin, J.) 941 (Elsevier Science, North-Holland Amsterdam, 1992).

18. Srinivasan, K. \& Painter, O. Linear and nonlinear optical spectroscopy of a strongly-coupled microdisk-quantum dot system. Nature 450, 862-865 (2007).

19. Englund, D., Faraon, A., Stoltz, N., Petroff, P. \& Vučković, J. Controlling cavity reflectivity with a single quantum dot. Nature 450, 857-861 (2007).

20. Chang, D. E., Sørensen, A. S., Demler, E. A. \& Lukin, M. D. A single-photon transistor using nano-scale surface plasmons. Nature Phys. 3, 807-812 (2007).

21. Carmichael, H. J., Kochan, P. \& Sanders, B. C. Photon correlation spectroscopy. Phys. Rev. Lett. 77, 631-634 (1996)

22. Thompson, R. J., Turchette, Q. A., Carnal, O. \& Kimble, H. J. Nonlinear spectroscopy in the strong-coupling regime of cavity QED. Phys. Rev. A 57, 3084-3104 (1998).

23. Meekhof, D. M., Monroe, C., King, B. E., Itano, W. M. \& Wineland, D. J. Generation of nonclassical motional states of a trapped atom. Phys. Rev. Lett. 76, 1796-1799 (1996).

24. Mielke, S. L., Foster, G. T. \& Orozco, L. A. Nonclassical intensity correlations in cavity QED. Phys. Rev. Lett. 80, 3948-3951 (1998).

25. Foster, G. T., Orozco, L. A., Castro-Beltran, H. M. \& Carmichael, H. J. Quantum state reduction and conditional time evolution of wave-particle correlations in cavity QED. Phys. Rev. Lett. 85, 3149-3152 (2000).

26. Birnbaum, K. M. et al. Photon blockade in an optical cavity with one trapped atom. Nature 436, 87-90 (2005).

27. Nußmann, S. et al. Vacuum-stimulated cooling of single atoms in three dimensions. Nature Phys. 1 , 122-125 (2005).

Supplementary Information accompanies this paper on www.nature.com/naturephysics.

\section{Acknowledgements}

We thank N. Syassen for early contributions. Partial support by the Bavarian PhD programme of excellence QCCC, the DFG research unit 635, the DFG cluster of excellence MAP and the EU project SCALA are gratefully acknowledged.

Author information

Reprints and permission information is available online at http://npg.nature.com/reprintsandpermissions. Correspondence and requests for materials should be addressed to G.R. 